\title{
LE CERCLE SÉMIOTIQUE DE GREIMAS
}

\section{GREIMAS' SEMIOTIC CIRCLE}

ERIC LANDOWSKI *

RÉSUMÉ : Décrire de l'intérieur le mode de fonctionnement du groupe de recherche sémiotique créé par A. J. Greimas à la fin des années 1960, analyser son évolution jusqu'aujourd'hui, saisir l'image qu'il a donné de lui-même dans son environnement académique et sur la scène intellectuelle, et par là chercher à mieux comprendre la nature et l'étendue de son influence, mais aussi à en expliquer les limites, tel est l'objectif de cet article, esquisse d'une socio-sémiotique de l'aventure « greimassienne».

MOTS CLEFS : Actant collectif. École de Paris. Histoire de la sémiotique. Greimas.

ABSTRACT: Describing the dynamics of the semiotic research circle created by A. J. Greimas in the late sixties, analysing its development up to now, capturing the image that the socalled Paris School of Semiotics has conveyed of itself on the intellectual scene, and thereby trying to understand not only the nature of its influence but also the factors which have contributed to limit its diffusion: such are the objectives of

* Centre National de la Recherche Scientifique - CNRS/Paris.

E-mail : eric.landowski@sciencespo.fr. 
this article, which aims at sketching a sociosemiotic survey of «Greimasian » experience.

KEYWORDS: Collective actant. Paris School. Greimas. History of Semiotics.

Toute rencontre est interrogation. Questionnement d'ordre existentiel ou simplement professionnel, il faut d'une manière ou d'une autre répondre de ce qu'on « est ». «Seriezvous donc poète ? romancier? ou peut-être philosophe? Rien de tout cela : sémioticien. - Mais encore ? peircien ou lotmanien ? - Ni l'un ni l'autre : greimassien ! A Aucun de ces trois adjectifs ne doitévidemment être pris comme une marque d'allégeance relevant de quelque culte de la personnalité : ce sont de simples étiquettes. Tout en perpétuant la mémoire des pères fondateurs - Charles S. Peirce (1839-1914), Youri M. Lotman (1922-1993), Algirdas J. Greimas (1917-1992) -, elles servent, entre initiés, à désigner les principaux courants théoriques entre lesquels les sémioticiens se répartissent de nos jours : trois familles d'esprit dialoguant entre elles, ou trois écoles rivales? Nous semble plus juste l'idée de cercles de pensée distincts et autonomes, et à certains égards complémentaires au sein d'une même discipline. C'est du moins ce que nous chercherons à justifier à propos du groupe de recherche "greimassien » en cherchant à décrire son évolution et son fonctionnement, et par là à comprendre la nature et l'étendue de son influence, mais aussi à en expliquer les limites. 


\section{Le fondateur}

Une réflexion sur le contenu théorique de ces courants n'étant pas l'objet du présent essai, indiquons tout au plus ce qui les distingue très globalement. Tandis que les « peirciens » développent une sémiotique interprétative articulée sur une philosophie du signe et que les « lotmaniens » se donnent pour objectif d'élaborer une sémiotique de la culture, les « greimassiens » ont l'ambition de construire une théorie générale de la signification qui permette de rendre compte des conditions d'émergence et des modes d'articulation du sens investi dans les discours, les pratiques et les objets de tous ordres. Ces trois variantes de la sémiotique, qui jouissent chacune aujourd'hui d'une large diffusion, s'enracinent dans des contextes historiques, intellectuels, sociaux et politiques qui, propres à leurs fondateurs respectifs, diffèrent entre eux du tout au tout.

Bien qu'unanimement reconnue de nos jours comme une contribution majeure à la tradition pragmatiste nordaméricaine, l'œuvre de Peirce fut, de son vivant, celle d'un penseur académiquement marginalisé, presque sans éditeur, sans disciple et, à quelques rares exceptions près, privé d'interlocuteurs. Sa philosophie, et a fortiori sa sémiotique, ne commenceront à être systématiquement publiées qu'à titre posthume à partir des années 1950. Pour de tout autres raisons, la sémiotique lotmanienne prend elle aussi naissance dans un environnement qui ne lui est guère propice. S'appuyant sur les travaux précurseurs des cercles linguistiques de Moscou et de Prague dont les bouleversements des années 20 et 30 avaient depuis longtemps entraîné la dispersion, c'est dans le climat idéologiquement hostile de l'Union Soviétique du second après-guerre, reléguée de Moscou à Tartu et sans beaucoup de 
contacts avec le monde extérieur, qu'elle parvient à se constituer. Par comparaison, la conjoncture qui préside à la naissance de l'entreprise greimassienne semble presque providentielle.

Rien ne permettait pourtant de le prévoir. Toute la première moitié de la vie de Greimas, né à Toula de parents chassés de Lithuanie pendant la première guerre mondiale (puis déportés en Sibérie à l'issue de la seconde), se caractérise en effet comme une suite de tragiques ballottements liés aux péripéties de l'histoire. Et c'est seulement au prix de l'exil, âgé déjà de près de quarante ans, qu'il trouve, une fois définitivement établi en France, la stabilité nécessaire à l'épanouissement de sa vocation de chercheur. Mieux que cela, il y trouve à vrai dire un contexte des plus favorables à la gestation du projet scientifique qui va être le sien. Car au milieu de l'effervescence intellectuelle qui règne dans le Paris de l'après-guerre commence à se dessiner un courant de pensée promis à bientôt reconfigurer le champ intellectuel français autour des principes constitutifs de ce qu'on appellera d'ici peu le structuralisme.

Greimas s'y voit d'emblée reconnu une place en tant que théoricien du langage et, plus largement, en tant que l'un des promoteurs d'une nouvelle épistémologie pour les sciences sociales dans leur ensemble. A ce titre, entre 1956 et 1966, il contribue à toutes les grandes revues où la génération nouvelle - qui vient de découvrir (ne fût-ce encore que fragmentairement) l'œuvre de Saussure - est en train de définir sa position à la fois par rapport aux approches phénoménologiques développées par la génération précédente et par rapport à la théorie marxiste (ainsi que, plus marginalement, vis-à-vis de la doctrine psychanalytique). $\mathrm{Au}$ long de cette décennie d'intenses débats, sa signature apparaît tour à tour dans Arguments (revue de philosophie 
politique fondée en 1956 par Edgar Morin, Roland Barthes et Jean Duvignaud), dans les Annales (organe créé par l'historien Lucien Febvre dès 1929 mais renouvelé à partir de 1951 en passant sous la direction de Fernand Braudel et Robert Mandrou), dans L'Homme (revue d'anthropologie que dirigent Emile Benveniste et Claude Lévi-Strauss et dont le premier numéro paraît en 1961) et même, chose plus inattendue de la part d'un défenseur déclaré de l'optique structurale, dans les Temps Modernes (fondés en 1947 par Jean-Paul Sartre et Maurice Merleau-Ponty) ${ }^{1}$.

C'est dire que la pensée du futur chef de file de la sémiotique de langue française, loin de pâtir du moindre isolement dans son milieu d'adoption parisien ni d'aucune forme de persécution, fut au contraire, au départ, intimement associée, en ce qu'elle présentait de plus novateur, à un vaste courant de réflexion collectif. Si à ce moment-là Greimas n'a encore rassemblé autour de lui aucun « cercle », aucun club de discussion, aucune équipe de collaborateurs, ni a fortiori aucune " école », il se trouve en revanche intégré dans une puissante famille d'esprit dont les principaux représentants l'accueillent (tel Claude Lévi-Strauss, qui de 1967 à 1969 l'héberge au Collège de France), le stimulent (tel Roland Barthes, alors son proche compagnon de travail) et le soutiennent (tel Charles Morazé à la Fondation de la Maison des Sciences de l'Homme).

La différenciation ultérieure des approches globalement recouvertes, un moment, par l'étiquette de "structurales ", mais aussi, en cette période de guerre froide, l'intransigeance dont Greimas dut faire preuve dans la défense de ses options

1 Soit, respectivement A. J. Greimas : 1956a ; 1956b ; 1958 ; 1963 ; 1966a (contribution à un dossier sur la notion de structure rassemblant, entre autres, des interventions de Lucien Sebag, Pierre Bourdieu et Charles Morazé ; texte réédité aussi dans Du sens). 
intellectuelles, morales et politiques alors qu'en se résignant à émigrer à l'Ouest il venait, d'une certaine façon, de passer d'un camp à l'autre, tout cela aboutit à ce que bientôt il n'en fut plus du tout de même pour lui. A tel point que tout le reste de sa vie son relatif isolement sur la scène intellectuelle française devait aller de pair avec la singularité de son projet théorique.

Cependant, entre son départ de Lithuanie (1945) - avec laquelle il ne rompra jamais les liens - et son installation définitive en France (1960), Greimas passe en Egypte, puis en Turquie, une longue période de transition marquée par la méditation, les lectures et quelques rencontres décisives. Maître de conférence à l'université d'Alexandrie, il y enseigne, de 1949 à 1958, l'histoire de la langue française. C'est là qu'il fait en particulier la connaissance de Roland Barthes. Partageant la même curiosité et les mêmes interrogations devant ce qui touche à la culture, à la littérature, à la langue, ils se lient d'amitié. A leurs échanges prennent part aussi le philosophe Charles Singevin et quelques autres personnalités qui joueront également un rôle dans le renouveau des sciences humaines et sociales en France. Sous l'impulsion de Greimas, un cercle de discussions régulières prend bientôt tournure, cercle informel et de nature interdisciplinaire soudé par le goût de la confrontation entre pensées innovatrices. A bien des égards, on a là, à Alexandrie, la préfiguration du mode d'existence d'un groupe de chercheurs tel que Greimas le rêvait et tel qu'environ vingt ans plus tard, à Paris, il parvint à le concrétiser : celui, comme il aimait à dire, d'un « club des égaux ». 
De fait, au cours des années 1970, c'est bien sous la forme d'une sorte de club fondé sur le volontariat, l'estime intellectuelle réciproque et un sens délicat de la convivialité que se constitue autour de lui, cette fois en vue d'une entreprise scientifique de longue haleine, une équipe de collaborateurs à la fois diversifiée en termes d'appartenances disciplinaires et unifiée par l'adhésion à un minimum épistémologique commun ainsi que par une exigence partagée de rigueur sur le plan conceptuel. Greimas n'exerçait aucun pouvoir et ne disposait d'aucune prébende à distribuer. A la différence de la plupart de ses collègues et de ses pairs, Français de souche, normaliens et agrégés, lui, Français d'adoption formé pour l'essentiel à l'étranger, ne faisait partie d'aucune de ces confréries, d'aucun de ces réseaux, si influents en France, que constituent les amicales, formelles ou informelles, d'anciens élèves des grandes écoles. Par ailleurs, s'astreindre, fûtce pour la bonne cause, à jouer comme beaucoup d'autres, avec application et persévérance, des rouages de l'institution académique dont il relevait était apparemment au-dessus de ses forces, ou en-dessous du seuil de son amour-propre. En conséquence, il n'appartint jamais à aucune clientèle ni ne fut affilié à aucun groupe d'influence. Ignoré qui plus est par les médias, à aucun moment ne lui fut à proprement parler attribué une place parmi les figures les plus en vue de l'intelligentsia parisienne. Atavisme peut-être venu de la sylvestre Suwalkija de ses aïeux (aux confins de la Pologne), il resta toujours plus ours que mondain ${ }^{2}$. Intransigeant, fier et obstiné : un pur Lithuanien! A Paris !

Rien de tout cela pourtant ne l'empêcha d'être, à sa manière, un rassembleur charismatique - un constructeur

2 Sur ces traits de la personne, en relation avec l'œuvre, cf. Landowski (2009), « Honoris causa ». 
d'« actants collectifs », selon son expression. Groupements éphémères ou relativement durables, informels ou, plus rarement, institutionnellement ancrés, à vocation fonctionnellement circonscrite (par exemple pédagogique ou éditoriale) ou d'ordre plus "politique ", il ne cessa, sa vie durant, de créer, moins autour de sa personne que de ses idées, des collectifs de toutes sortes - parfois, il est vrai, uniquement sur le papier. Boy scout comme il était de règle à son âge un peu partout dans l'Europe des années 30, il ne se contenta pas de se plier à l'esprit d'embrigadement qui était dans l'air du temps mais voulut, paraît-il, refonder les liens soudant la troupe ${ }^{3}$. De même, un peu plus tard, sous l'occupation allemande, résistant, il chercha, dans la clandestinité, à constituer un parti. Et vers la fin de sa vie, dans l'espoir de rallier et de canaliser l'énergie de ses compatriotes, il s'employait à dessiner à leur attention la figure idéale de l'actant-nation Lithuanie, en particulier dans une longue lettre adressée au président de la République tout juste redevenue indépendante ${ }^{4}$. Entretemps, dans l'exercice de son métier de chercheur et, complémentairement, de professeur, Greimas, en théoricien à la fois convaincu de la nécessité du travail en équipe et soucieux de promouvoir la diffusion de sa pensée sémiotique, s'était inlassablement employé, à partir des années 1960, à faire vivre autour de lui groupes de travail et espaces de réflexion.

Voir l'article de Thomas F. Broden (2011).

4 Cf. «Pro memoria : ponui Lietuvos Respublikos Prezidentui Vytautui Landsbergiui », lettre publiée à titre posthume par la revue Baltos lankos (GREIMAS, 1997). Témoigne du même esprit rassembleur et militant le petit volume La Lithuanie, un des pays baltes (GREIMAS, A. J. ; ŽUKAS, S., 1993), présentation historique en même temps que plaidoyer politique rédigé en collaboration avec Saulius Žukas et publié en 1993 chez l'éditeur Baltos lankos (dans les deux langues). Dans le même sens, cf. le recueil d'interventions dans la presse dissidente établi par Arūnas Sverdiolas (1998) sous le titre Gyvenimas ir galvojimas: la vie et la réflexion. 


\section{Portrait de groupe}

Le premier, le principal de ces espaces aura été celui de son séminaire de "Sémantique générale » à la $\mathrm{VI}^{\mathrm{e}}$ section de l'Ecole Pratique des Hautes Etudes (devenue par la suite Ecole des Hautes Etudes en Sciences Sociales). On lui a vu prendre des formes diverses au fil du temps.

La phase initiale paraît rétrospectivement atypique car le mode de fonctionnement du séminaire n'est pas encore celui que demande la construction d'un projet commun. Il s'agit plutôt d'un espace de rencontre - plus exactement, de confrontation - entre des esprits qui n'ont que peu de choses en commun et dont chacun s'apprête à suivre au plus vite son propre chemin indépendamment de tous les autres. Ce séminaire première manière a donc beau être prestigieux et témoigner de l'aura de Greimas - de semaine en semaine y défilent bon nombre des futurs représentants du French thinking -, il ne doit pas faire illusion : à ce stade, il n'existe pas encore de « cercle sémiotique greimassien ». Ni le projet de narratologie, alors embryonnaire, que Gérard Genette vient y présenter, ni, par exemple, la pragmatique qu'y esquisse Oswald Ducrot, ni même l'approche mi-sémiologique minarratologique qu'y défend Christian Metz en vue d'une théorie du cinéma, ni, encore moins, la " séméiotique » dont Julia Kristeva (déjà partie prenante en première ligne du groupe Tel Quel) délègue l'exposition à un de ses proches, ne s'inscrivent dans une perspective qu'on puisse appeler « greimassienne». Pas plus que les travaux d'un Brémond, d'un Todorov ou encore d'un Tomas Pavel, autres participants de l'époque. Que tous ces auteurs se soient à un moment donné aguerris par la confrontation, en général âpre, avec Greimas, cela n'en a évidemment pas fait des « greimassiens »! Pour la plupart 
d'entre eux, ce fut même tout le contraire, et les clivages apparus en ces circonstances ne firent ensuite que se confirmer, à commencer par celui, épistémologiquement et stylistiquement le plus patent et le plus profond, entre « greimassiens » et « tel quelliens »: d'un côté, une conception de la recherche tout empreinte de classicisme, visant l'anonymat et la systématicité de la « science », privilégiant pour cela la cohérence et la clarté dans l'ordre conceptuel, de l'autre, par comparaison, un style de pensée « baroque » autorisant tous les éclectismes et qui allait déboucher dans un subjectivisme hautement revendiqué 5 .

Mais la crise de 1968 apporte une nouvelle génération de chercheurs et, avec elle, un autre mode de fonctionnement du séminaire. Certes, il arrive encore, de temps à autre, que telle ou telle figure marquante (autre que les précédentes) vienne débattre des questions que pose le développement du projet sémiotique. Témoins extérieurs d'autant plus utilement critiques qu'ils sont dans l'ensemble sympathisants de l'entreprise, Michel de Certeau, Paul Ricœur, Louis Marin, Georges Kalinowski, Bernard Pottier, Bruno Latour, Paul Zumthor, Umberto Eco, Italo Calvino, entre autres, font partie de ces visiteurs épisodiques à la fois renommés au dehors et familiers en ces lieux. Cependant, l'ordinaire des réunions se déroule sous la forme plus modeste, moins spectaculaire ou plus austère, de discussions à caractère non pas fermé mais endogène. Ce dont on débat, ou plus exactement ce à quoi on travaille ensemble, c'est à la consolidation et $\mathrm{au}$ développement du projet sémiotique, sous-entendu greimassien, et à cela seul. Pendant de nombreuses années, les discussions s'organisent autour de présentations systématiques du responsable du séminaire, qui teste de cette

5 Sous une forme moins exacerbée, une opposition du même genre finira d'ailleurs par réapparaître, bien plus tard, à l'intérieur même du groupe greimassien. Cf. Leone (2013). 
manière ses travaux en cours, avant leur publication'. Plus tard, davantage de place sera donnée aux interventions de ses collaborateurs en tant que parties prenantes pleinement associées au développement de la théorie. Et à partir de ce moment, afin de regrouper les efforts tout en les orientant, les travaux s'articulent à l'intérieur de thématiques renouvelées d'une année à la suivante et débouchant, dans les meilleurs des cas, sur quelque volume rédigé à deux ou à plusieurs ${ }^{7}$. « Il faut organiser la démocratie », ironisait notre stratège de la recherche à propos de ses propres stratégies.

A côté du séminaire gravitaient d'autres regroupements visant des objectifs plus spécifiques. - C'étaient d'abord les « ateliers », soit une demi-douzaine environ de petites équipes à fonction à la fois heuristique et didactique. Chacun réunissait autour d'un responsable les spécialistes d'un champ d'étude déterminé : sémiotique des discours scientifiques (Françoise Bastide), religieux (Jean Delorme), ou littéraires (Denis Bertrand), et aussi, à part égale avec les études textuelles mais privilégiant l'analyse des objets et des pratiques, les ateliers de sémiotique de l'espace (Manar Hammad), d'ethnosémiotique (Joseph Courtés) et de socio-sémiotique (Eric Landowski), sans oublier celui de sémiotique visuelle animé par Jean-Marie Floch, où, en quelques années, s'élabora une problématique inédite en matière d'approche des images (de la peinture à la publicité en passant par la photographie) ${ }^{8}$. -

6 Ainsi notamment de son Maupassant: la sémiotique du texte (GREIMAS, 1976) ainsi que de son ultime ouvrage, De l'Imperfection (GREIMAS, 1987).

7 Soit, issus l'un et l'autre des travaux du séminaire, A. J. Greimas et J. Fontanille (1991) ; A. J. Greimas et E. Landowski (1979), avec des contributions, entre autres, de J. Geninasca et J.-Cl. Coquet.

8 Cf. J.-M. Floch (1985), Petites mythologies de l'œil et de l'esprit, synthèse des travaux de l'atelier et ouvrage fondateur de la sémiotique figurative et plastique d'inspiration greimassienne. 
C'était aussi l'équipe éditoriale, un temps à la tête de deux collections de livres, l'une en français, l'autre en anglais ${ }^{9}$, et, plus durablement, responsable surtout de la revue du groupe, les Actes Sémiotiques. Tâche de longue haleine puisque cette revue créée en 1978 sous une forme des plus modestes (initialement un simple Bulletin d'information de quelques pages ronéotées, mais accompagné dès 1979 d'une série de Documents à caractère monographique édités par l'Institut National de la Langue Française, puis, à partir de 1989, par les Presses de l'université de Limoges) est toujours en activité ${ }^{10}$. Non seulement les Actes sont aujourd'hui la seule revue de sémiotique en France mais ils restent dans le monde entier une publication de référence pour la plupart des sémioticiens d'obédience structurale. - C'était encore l'Association pour le Développement de la Sémiotique, chargée de missions diverses concernant la gestion de la recherche et qui fut un moment présidée par Paul Ricœur. - Et c'était enfin, subsumant tout cela, seule entité formellement dotée d'un statut institutionnel, le Groupe de Recherches Sémio-linguistiques, unité du Centre National de la Recherche Scientifique en même temps que laboratoire de l'Ecole des Hautes Etudes en Sciences Sociales.

9 Outre l'ouvrage précité, une quinzaine de volumes sont parus dans les collections « Actes Sémiotiques » et « Semiotic Crossroads » publiées de 1984 à 1990 par Hadès-John Benjamins (Paris-Amsterdam). Citons parmi eux J. Fontanille, Le savoir partagé (1987), H. Parret, Le sublime du quotidien (1988), ainsi que deux panoramas généraux sur les travaux du groupe : E. Landowski et al., Sémiotique en jeu. A partir et autour de l'ouvre d'A. J. Greimas (1987), et P. Perron (Org.), Paris School Semiotics (2 vol., 1989).

10 Depuis 2007 les Actes Sémiotiques sont accessibles « en ligne » : <http://epublications. unilim. fr/revues/as/index.php>. 
Des procédures de construction de toutes ces instances où la participation de chacun prenait son sens en concourant à la réalisation d'un objectif commun, Greimas fit un jour la théorie ${ }^{11}$. Comment articuler la libre initiative individuelle et les exigences d'une entreprise d'intérêt collectif ? L'accomplissement de soi n'est-il concevable que dans la transcendance d'un Nous? C'est peut-être ce genre de questions que cherchait déjà à résoudre le jeune scout des années $30 \ldots$ En tout cas, le modèle que Greimas mit au point quarante ans plus tard tient encore à présent et va pouvoir ici même nous servir. Il permet de penser le passage entre ce qui est de l'ordre de l'unité et ce qui définit une totalité une collectivité quelconque - en fonction de ses divers modes d'existence possibles.

Une « unité » peut être pensée soit comme intégrale, à la manière d'un individu dans son irréductible singularité, soit comme de caractère partitif. Tout individu, si singulier soit-il, partage en effet nécessairement quelques traits avec certains autres, et à ce titre fait virtuellement partie d'un ensemble. La forme d'une collectivité peut, par suite, être à son tour pensée de deux manières. Ou bien d'un point de vue quantitatif, comme une totalité elle-même " partitive » obtenue par la simple juxtaposition d'unités individuelles singulières mais à tel ou tel égard semblables. Ou bien qualitativement, comme une totalité à caractère de nouveau "intégral », formant un seul bloc, une seule «personne morale » en dépit de sa pluralité interne : ainsi de la nation, entité politique réputée une et indivisible. Une philosophie de l'histoire en même temps qu'une éthique personnelle, située aux antipodes de l'individualisme «post-moderne », est presque explicitement

11 A l'occasion d'un travail sur le droit : " Analyse sémiotique d'un discours juridique » (GREIMAS, 1971). 
sous-jacente à cette construction : c'est seulement moyennant l'acceptation et même la quête de son propre dépassement au profit de la totalité d'arrivée, à caractère transcendant et englobant, que l'unité de départ - le moi, cette " forme évanescente $»^{12}$ - prend sens et peut trouver une justification à sa problématique existence.

Si ce schéma vise d'abord à rendre compte des processus de construction des formations collectives, il permet aussi de prévoir les modalités de leur dissolution. De ce double point de vue, il éclaire bien le destin de la plupart des collectifs suscités par Greimas, et en particulier celui du plus important d'entre eux en termes fonctionnels et institutionnels puisque tout - recherche, publications, enseignement, y compris le séminaire - y était rattaché, à savoir feu le « GRSL », Groupe de Recherches Sémio-linguistiques de l'Ecole des Hautes Etudes.

Il s'était d'abord constitué de bric et de broc, vers la fin des années 60, par la simple juxtaposition de vocations individuelles éparses. Puis il fut rapidement unifié sous la houlette de son « directeur» - plus exact serait de dire sous le charme d'un " Destinateur » nullement enclin à fixer des règles mais capable de donner du sens et de la valeur à une action menée en commun. La consolidation progressive de la théorie, niveau après niveau (du plus profond au plus superficiel), aussi bien que les redéfinitions successives de son objet (du textuel à l'interactionnel) et que l'extension progressive de ses terrains d'investigation empirique (champ

12 Formule chère à Greimas, qui l'avait empruntée à Michel de Certeau. 
par champ), étaient effectivement conduites, pour une large part, à la manière d'actions concertées. D'où le titre d'Actes sémiotiques que nous avions voulu donner à la revue, et qui plut à Greimas pour sa valeur connotative. De fait, les forces des uns et des autres étaient mobilisées tour à tour, sur un mode assez impératif, dans de véritables campagnes d'exploration ou de conquête conceptuelles dont les résultats seraient ensuite, si possible, intégrés au modèle théorique commun. Avec parfois, en attendant de nouveaux assauts, des replis stratégiques face aux obstacles rencontrés lors de tentatives d'avancée qui étaient souvent plus que risquées.

C'est comme cela que pendant près de vingt ans ce groupe pensa et produisit, chose rare en France dans le monde des sciences humaines et sociales, sous la forme d'une vraie petite totalité intégrale. Certes, ni l'hétérogénéité des formations ni les différences d'orientation philosophique ou théorique des uns et des autres ne s'étaient miraculeusement effacées. Pas plus que les disparités statutaires, les hiérarchies, les rivalités et les jalousies. Ni les ambitions personnelles. Mais tout cela se trouvait tendanciellement transcendé dans la poursuite d'un projet intellectuel assez mobilisateur pour exercer sur tous un pouvoir puissamment fédérateur. Etant donné qu'en toutes choses il y a malgré tout "des hauts et des bas », il pouvait bien sûr arriver que quelque faiblesse menace de faire succomber l'un ou l'autre à la tentation de la partitivité. Greimas n'avait alors qu'un remède, mais efficace, à savoir cette exhortation aussi virile et généreuse qu'insidieusement compulsive : «Elevez le regard !» Et du coup les affaires (sinon la vie même) reprenaient un sens. C'était la belle époque des années 70-80.

Ensuite de quoi, dès avantla mort de son fondateur, il fallut malheureusement se résigner à voir ce chef d'œuvre d'entente 
fiduciaire régresser en quelques années à l'état d'une totalité partitive juxtaposant des sous-groupes rivaux. L'existence de clivages internes n'était pas en elle-même une nouveauté. Au contraire, l'explicitation des désaccords et la critique des uns par les autres avaient toujours fait partie de la règle du jeu. Cela constituait même un des principaux moteurs de la dynamique intellectuelle du groupe ${ }^{13}$. Mais il n'en fut plus de même à partir du moment où l'expression des divergences théoriques commença à servir de justification pour la formation de clans adverses et de clientèles particulières. Inexorablement, le club des égaux, cette république de jeunes chercheurs libres et insoucieux, était en train de se transformer en une sorte de société féodale fragmentée, soumise à des jeux d'alliances tactiques entre mandarins, sous-mandarins (autres mots de Greimas) et leurs affidés, les uns comme les autres désormais d'autant plus préoccupés de leur avenir et de leur carrière que, dans le climat de crise économique qui venait de s'installer, les postes et les crédits tendaient à se faire plus rares. Après l'apogée, c'était le commencement de la fin... Non pas celle de la « sémiotique greimassienne » en tant que projet théorique mais celle du « cercle greimassien » en tant que groupe de pairs, de collaborateurs solidaires et d'amis.

L'éclatement du groupe en une série de tendances autonomes, d'unités partitives qui s'éloignaient les unes des autres à qui mieux mieux comme par entropie ne suffit pas toutefois à faire perdre aux ex-associés l'air de famille qu'ils avaient acquis du temps où le sentiment d'un intérêt collectif les unissait. Bien que devenus entre eux, à peu de choses près, des étrangersou même, pour certains, des frères ennemis, ilsrestaient

13 En témoigne le second tome du Dictionnaire de Greimas et Courtés (1986), où les contributions d'une vingtaine de collaborateurs font apparaître l'hétérogénéité des positions défendues à l'intérieur du groupe. 
tous, aux yeux du monde extérieur, des " greimassiens ». Et de fait, le gros de la troupe continua d'assumer cet héritage, mais en le modulant selon des formes de plus en plus diversifiées. C'est ainsi qu'à la sémiotique greimassienne d'antan, vue sans doute trop idéalement comme « une et indivisible » (mais cela aussi répondait à une des stratégies de son fondateur ${ }^{14}$ ), succéda le déploiement d'approches qui tout en continuant de se situer globalement à l'intérieur du même cadre épistémologique se posaient, désormais explicitement, comme autant d'alternatives concurrentes en vue de son renouvellement, de son prolongement ou de son enrichissement. Aujourd'hui, les approches connues sous le nom de sémiotique " tensive », de sémiotique « modulaire » et de « socio-sémiotique » constituent les courants post-greimassiens à large diffusion répondant le mieux à ce positionnement : diversification et renouveau, mais sans reniement du passé ni rejet de l'acquis, sans rupture épistémologique ni psychodrame œdipien ${ }^{15}$.

Pour quelques autres en revanche, passer pour greimassien ou même ex-greimassien devint une sorte de stigmate porté à contre-cœur. Pour exister, il leur fallait non seulement, comme on dit, "tuer le père ", mais surtout se désolidariser publiquement de sa tribu dans l'espoir d'être reconnu pour soi-même en tant qu'unité intégrale et non plus étiquetté comme partie d'un tout, disciple d'un maître, ex-membre d'une équipe. Coquetterie compréhensible mais qui se révéla contreproductive et même, un court moment, catastrophique. A tel point que la fin de cette histoire aurait pu ramener à son point de départ: des égaux à l'Ego, au chacun

14 Cf. sur ce point J. Geninasca (1994), « Et maintenant ?».

15 Cf. respectivement J. Fontanille et Cl. Zilberberg (1998); J. Geninasca (1997) ; E. Landowski (2004). 
pour soi, au pur « subjectal $»^{16}$. Il s'en est fallu de peu. Mais une fois passée la crise des années 90, les forces cohésives l'emportèrent de nouveau, en sorte qu'en définitive l'esprit de l'entreprise survécut pour l'essentiel, sous de nouvelles formes, à l'autonomisation de ses participants.

Les lieux où se manifestent cette persistance et ce renouvellement sont multiples et divers. C'est en particulier la revue déjà mentionnée, les Actes Sémiotiques, dont les responsables, tout en veillant à maintenir le cap initialement fixé - avancer aussi loin que possible dans le cadre rigoureux de l'option théorique structurale - , cherchent à promouvoir, contre l'esprit d'Ecole, une sémiotique intellectuellement créative, ouverte aux courants de pensée contemporains et en prise sur les problèmes de la vie d'aujourd'hui. Ce sont aussi les collections de livres, celles des Presses Universitaires de France et des Presses de l’Université de Limoges («Formes sémiotiques », "Actes sémiotiques », "Semiotica viva »). C'est encore le séminaire général, qui continue depuis des décennies d'offrir un lieu de discussion. Et ce sont surtout les centres de recherche qui ont essaimé un peu partout à l'étranger, spécialement en Italie et au Brésil, accompagnés de leurs propres revues, maisons d'éditions, associations et curriculums universitaires.

\section{Le regard des autres}

Cesquelques aperçus relatifsà la naissance etàl'évolution du groupe, à ses métamorphoses vécues de l'intérieur, laissent de côté bien d'autres éléments dont il faudrait aussi tenir

16 Sur la sémiotique « subjectale », cf. J.-Cl. Coquet (1984), Le discours et son sujet. 
compte pour comprendre la nature particulière, et les limites, de son impact sur l'espace environnant. Comment ce groupe s'est-il présenté au dehors? En le voulant ou sans le vouloir, quelle image a-t-il donné de lui-même, en premier lieu dans son contexte le plus proche, le milieu intellectuel et d'abord universitaire français?

Revenons un instant en arrière. En 1982, alors que n'existait aucun manuel de sémiotique et que les débutants en étaient réduits à la lecture de Sémantique structurale, ouvrage fondateur mais d'accès très difficile, ou à celle, non moins ingrate, d'un Dictionnaire de sémiotique qui semblait avoir été fait pour tester la cohérence de la théorie plutôt que pour initier quiconque à son emploi ${ }^{17}$, parut chez un éditeur de bonne diffusion un petit volume qui se présentait comme le premier « reader » en la matière : Sémiotique. L'Ecole de Paris $^{18}$. Ce titre aussi pompeux qu'inattendu avait semblé commercialement prometteur au service de marketing et, à ce qu'on peut supposer, académiquement flatteur à l'organisateur du recueil. L'avis des huit contributeurs n'avait pas été demandé. Celui de Greimas non plus. Mais comme il avait pour principe de ne pas décourager les bonnes volontés ("ne pas désespérer Billancourt», disait-on à l'époque), il ne refusa pas, et même, vu son ironie coutumière, s'amusa de se voir ainsi reconverti en maître d'école. Jamais en revanche, pour désigner la petite communauté qu'il avait rassemblée autour de lui, il ne reprit cette dénomination à son compte. Le syntagme « Ecole de Paris » ne figure dans aucun de ses écrits, et en vingt-cinq ans de proche collaboration nous ne l'avons

17 Soit, respectivement, A. J. Greimas (1966a) et A. J. Greimas et J. Courtés (1979).

18 J.-Cl. Coquet (1982), Sémiotique : L’Ecole de Paris, avec les contributions de M. Arrivé, Cl. Calame, Cl. Chabrol, J. Delorme, J.-M. Floch, C. Geninasca, P. Geoltrain, E. Landowski. 
pas une seule fois entendu le prononcer, sauf par dérision.

Il y avait à cela de bonnes raisons. La plus élémentaire tenait à ce qu'il n'est ni socialement de bon goût ni sémiotiquement de bonne règle de s'auto-glorifier. La consécration d'un groupe en tant qu'« Ecole » constitue une sanction qui de toute évidence ne peut provenir que d'un jugement extérieur. Se l'octroyer comme ce fut le cas, c'était se satisfaire d'un flatus vocis. C'était effectuer un acte performatif par avance manqué faute de la compétence requise puisque la seule instance habilitée à conférer une reconnaissance dans l'ordre scientifique, c'est la communauté scientifique, c'est-à-dire les autres. Il est vrai que malgré cela, les années passant, l'expression « Ecole de Paris » est entrée dans l'usage. Il n'en reste pas moins qu'elle allait exactement à contrepied de ce qui constituait, pour les membres de ce groupe, la raison même de leur être-ensemble : la poursuite d'une aventure intellectuelle commune. Car une Ecole, c'est d'abord une école, sans la majuscule : non pas une aventure partagée mais une institution chargée de transmettre un savoir constitué. Or ni Greimas ni la plupart de ses proches ne prenaient la sémiotique pour une science achevée. Elle restait un projet en construction, engageant une démarche à vocation scientifique. Ce n'étaient pas là des réserves de modestie mais l'expression d'une conception précise de la recherche, vue comme une suite d'avancées, d'homologations et de réexamens critiques appelant toujours de nouveaux dépassements. Et c'étaient en même temps autant d'avertissements contre la tentation du dogmatisme. - Ils n'ont pas été entendus. S'est au contraire répandue peu à peu une tournure d'esprit aussi scolaire que sectaire qui fit bientôt prendre les moindres formules du « maître » pour autant de 
paroles d'évangile ${ }^{19}$.

A cela tiennent aussi bien l'image négative du groupe en tant que tel, que le succès, auprès de deux camps diamétralement opposés mais qui se présupposentl'un l'autre, de l'étiquette d' «Ecole » (« de Paris ») qu'il s'était autocollée. Parmi les greimassiens, elle ne pouvait que combler d'aise les intégristes qui se croyaient les dépositaires d'un savoir indépassable et donc intangible. Et les anti-greimassiens eurent dès lors beau jeu de la leur renvoyer ironiquement pour dénoncer le caractère borné de leur adversaire : "Bien sûr que les greimassiens forment une école : que font-ils en effet depuis trente ans si ce n'est répéter inlassablement les leçons de leur "cher maître" ? ». Cercle, club ou tout simplement « groupe »-versus « Ecole »-, ce n'est donc pas là un choix anodin. Chacun de ces labels traduit différemment l'idée que les membres d'une collectivité se font de leur propre mode de participation. Etl'idée qu'ils s'en font ne peut pas être sans effet sur la vision qu'en ont les autres, au dehors. En l'occurrence, l'image d'une secte infatuée d'elle-même et réfractaire à tout échange est l'exacte reproduction en miroir de celle qu'une partie (minoritaire peut-être mais particulièrement en vue) des greimassiens se sont eux-mêmes donnée de leur groupe d'appartenance.

Mais cette psycho-sociologie ne suffit pas à expliquer tous les malheurs du groupe. Des facteurs conjoncturels ont aussi joué en sa défaveur. Autant en effet l'option pour une démarche scientifique "dure », armée de concepts, de

19 Ainsi de plusieurs « carrés sémiotiques », notamment celui dit de la "véridiction », ou celui des « quatre éléments » tels qu'articulés chez Maupassant et qu'on a voulu prendre pour un modèle de portée universelle ; ou de l'emprunt circonstanciel, à Wittgenstein, de l'expression «formes de vie », aussitôt adoptée comme une sorte de mot de passe entre fidèles et initiés ; ou encore de cette formule prononcée au fil d'une conférence : « Hors du texte, point de salut », interprétée comme un oukase fixant ad aeternam les limites de la sémiotique orthodoxe. 
modèles et d'un métalangage propre, avait dans un premier temps placé le projet sémiotique au cœur de l'épistémé du moment, autant, une fois passée la vogue structuraliste, la persistance sur cette même ligne allait mécaniquement reléguer le groupe dans une position à contrecourant. Continuer comme si de rien n'était à cultiver ce qui était de plus en plus considéré au dehors comme un jargon ésotérique, soupçonné qui plus est de ne recouvrir aucun contenu à force de ne renvoyer qu'à lui-même, cela aussi allait fortement contribuer à la marginalisation du cercle devenu " Ecole ». En France, sciences exactes mises à part, un discours un peu baroque, relativement hermétique mais fleuri et prêtant à une lecture « plurielle » (à la Barthes ou à la Tel Quel) vaut mieux auprès du public et des médias qu'un métalangage pur et dur, conceptuellement univoque mais imperméable à l'intuition. Si bien que tout en étant victime, comme toutes les sciences sociales, du mouvement de reflux qui suivit le tournant culturel de 1968, la sémiotique se trouva bientôt attaquée de deux côtés. Par les traditonnalistes qui l'avaient jugée dès le départ insupportablement scientiste et la taxaient à la fois d'« excès de rigueur » dans la démarche et d'une propension, au contraire laxiste, à l'« abus du jargon » dans les écrits. Et par la vague nouvelle des « postmodernes » enclins à voir dans tout projet de modélisation une forme de positivisme dépassé. En bonne logique, la critique des uns et des autres se concentrait sur ce que les sémioticiens considéraient, à l'inverse, comme la condition même d'une théorie cohérente et d'une méthodologie opératoire : leur métalangage.

Hélas, ni la théorie ni la méthode n'étaient plus au goût du jour! 
Mais la diffusion d'une pensée ne se mesure pas uniquement en termes de popularité. Paradoxalement, d'ailleurs, certaines des raisons mêmes de l'impopularité du groupe - sa grande exigence intellectuelle, par exemple expliquent peut-être aussi, dans l'autre sens, son influence en profondeur et à long terme. Il est vrai qu'en lui-même, le métalangage sémiotique construit pour rendre compte des conditions d'émergence et de saisie du sens constitue un bloc conceptuel si compact qu'hormis quelques greimassiens des plus orthodoxes personne ne « parle sémiotique » ni, a fortiori, " greimassien » (alors que dans un domaine proche, le «parler lacanien » ne semble pas l'apanage exclusif des analystes). Cela n'a cependant pas empêché la diffusion, par des voies souvent extérieures aux circuits académiques, de la pensée sous-jacente à ce vocabulaire. Car le mode de pénétration de la sémiotique greimassienne est un peu à l'image de son objet, le sens, chose invisible en surface et néanmoins partout présente, sous la surface des textes, des discours, des objets et des pratiques.

C'est ainsi que, de l'anthropologie à la philosophie du droit ou à la psychiatrie, de l'histoire de l'art aux études stratégiques (y compris militaires), ou encore, del'architecture aux sciences de la communication, au marketing et au design, le mode de raisonnement sémiotique a inspiré une multitude de chercheurs ayant affaire dans leur domaine propre à des problèmes de signification, qu'il s'agisse de l'analyse ou de la construction d'objets faisant sens ${ }^{20}$. De manière plus diffuse, l'esprit de la sémiotique greimassienne guide aussi beaucoup de sympathisants qui, ayant eu l'occasion de se familiariser par la lecture ou l'enseignement avec la problématique de

20 Cf., parmi bien d'autres, Cl. Calame et M. Kilani (1999); B. S. Jackson (1999); J.-P. Klein et I. Darrault (2007); O. Calabrese (1984); J. Ciaco (2013) ; A. Joxe (1991). 
la discipline mais n'ayant pas eu par la suite de raison d'en faire leur principal outil de travail, tirent intellectuellement profit du regard qu'elle leur a appris à projeter sur le monde en général. Et entre ce genre de compagnonnage à distance et les formes de coopération interdisciplinaire en règle, existe aussi une voie moyenne. C'est en Italie qu'on en trouve la meilleure illustration. Réussissant à faire usage des concepts de la discipline sans s'asservir à son lexique, à mettre les modèles abstraits au service d'une réflexion en prise sur la vie et à trouver un style d'écriture proche de l'essaiysme mais qui n'implique ni reniement ni oubli du souci de scientificité, ce sont en effet les sémioticiens italiens qui sont le mieux parvenus à faire sortir la discipline de son ghetto. Publiant dans des revues et chez des éditeurs à vocation généraliste, ils ont su la faire admettre comme une des voix appelées à exprimer une position critique sur la scène intellectuelle au sens le plus large ${ }^{21}$.

Le cercle s'est ainsi ouvert très au-delà de ses propres limites, au point de contredire à peu près tous les stéréotypes auxquels tient sa réputation mitigée. Vue d'ordinaire comme une problématique cantonnée dans l'analyse des textes littéraires (ce qui n'était pas tout à fait faux à l'origine), la sémiotique greimassienne n'a en fait cessé de s'affranchir du texte. Depuis plus de trente ans, la réflexion théorique porte sur les niveaux sous-jacents que présuppose la production et la saisie d'effets de sens attachés à des objets indifféremment verbaux ou non verbaux. Et l'attention se concentre aujourd'hui sur l'analyse des configurations d'ordre sensible ainsi que des processus d'ordre interactionnel dans le cadre desquels le monde fait sens, donnant lieu à l'apparition de

21 Cf., entre autres, P. Fabbri (2004) (chroniques initialement parues dans le quotidien L'Unità) ; G. Marrone (2011 ; 2012); M. Leone (2014). 
nouvelles « formes de vie $»^{22}$.

Ces évolutions obligent à revoir aussi les limites temporelles que beaucoup voudraientassignerà la sémiotique, spécialement greimassienne. Elle ne se réduit pas à un vieux souvenir des années pré-68! Il est vrai que l'ouvrage à partir duquel toute l'aventure a commencé, Sémantique structurale, est paru en 1966. Ce livre eut à l'époque un tel retentissement que Greimas reste, dans la mémoire de la plupart, « l'auteur de Sémantique structurale » et que dans beaucoup de milieux académiques la suite n'a jamais été prise sérieusement en considération, pas plus les travaux ultérieurs de l'auteur que ceux de son entourage. Pourtant, structurale ou post-structurale, la sémiotique a continué ! Et tout en restant fidèle à une base épistémologique minimale (qui laissait d'ailleurs grande latitude à l'esprit d'invention), elle a énormément changé. Le dernier Greimas, constructeur d'une sémiotique du sensible, n'a que peu à voir avec celui des premières années ${ }^{23}$. Quant à ce qui est du travail de ses successeurs, les nouvelles problématiques de la tensivité, de la saisie esthésique ou des régimes de sens dans l'interaction, auxquelles nous faisions allusion plus haut, ont refondé le cadre même de la réflexion, non seulement par rapport à la perspective sémantique du livre de 1966 mais aussi par rapport à celle, déjà bien différente, de la grammaire narrative qui dominait dix ans plus tard et que résume le classique Dictionnaire de 1979.

Enfin, dernière image à rectifier pour les mêmes raisons, celle de la sémiotique greimassienne comme article de Paris. Certes, c'est à deux pas de la Seine que le groupe s'est

22 Cf. E. Landowski (2005) ; A. C. de Oliveira (2013) ; J. Fontanille (2015).

23 Voir sur ce point A. J. Greimas (1987). 
constitué. Mais la plupart de ses membres n'ont jamais été parisiens que par intermittence, par accident ou par adoption. Le séminaire des belles années était composé aux trois quarts d'étudiants étrangers et de collègues venus de tous les continents. Une fois repartis, ils ont fondé leurs propres cercles dans leurs pays. En sorte que si c'est bien en France que cette sémiotique-là est née, c'est en tant que produit d'exportation qu'elle s'est ensuite développée. Et aujourd'hui plus que jamais, de Bologne à São Paulo mais aussi, hors de la " romanité », de Vilnius à Téhéran, ou de Meknès et d'Oujda à Istanbul, l'avenir du projet intellectuel greimassien se joue sur le plan international, loin du Quartier Latin.

\section{RÉFÉRENCES}

BRODEN, T. F. Towards a Biography of A. J. Greimas. Lituanus: The Lithuanian Quarterly Journal of Arts and Sciences, 57 (4), 2011.

CALABRESE, O. Il linguaggio dell'arte. Milan : Bompiani, 1984.

CALAME, Cl.; KILANI, M. La fabrication de l'humain dans les cultures et en anthropologie. Lausanne : Payot, 1999.

CIACO, J. A inovação em discursos publicitários: elementos de comunicação, semiótica e marketing. São Paulo : Estação das Letras e das Cores, 2013.

COQUET, J.-Cl. Le discours et son sujet. Paris : MéridiensKlincksieck, 1984.

. (Org.). Sémiotique : L'Ecole de Paris. Paris : Hachette, 1982.

FABBRI, P. Segni del tempo. Rome : Meltemi, 2004. 
FLOCH, J.-M. Petites mythologies de l'œil et de l'esprit. Paris-Amsterdam : Hadès-Benjamins, 1985.

FONTANILLE, J. Le savoir partagé. Paris-Amsterdam : HadèsBenjamins, 1987.

. Formes de vie. Liège : Presses de l'Université de Liège, 2015.

FONTANILLE, J.; ZILBERBERG, C. Tension et signification. Liège : Mardaga, 1998.

GENINASCA, J. La Parole littéraire. Paris : Presses Universitaires de France, 1997.

. Et maintenant? Documents de travail, Urbino, CISL, $n$. 230 [rééd. in LANDOWSKI, E. (Org.). Lire Greimas, Limoges, Pulim, 1997], 1994.

GREIMAS, A. J. Pro memoria : ponui Lietuvos Respublikos Prezidentui Vytautui Landsbergiui. Baltos lankos, n. 8, 1997. . De l'Imperfection. Périgueux : Fanlac, 1987.

. Maupassant: La sémiotique du texte. Paris : Seuil, 1976. Analyse sémiotique d'un discours juridique. Documents de travail, Urbino, CISL, n. 7 [rééd. in A. J. Greimas. Sémiotique et sciences sociales. Paris, Seuil, 1976], 1971.

. Structure et histoire. Les Temps Modernes, n. 246, nov., 1966a.

. Sémantique structurale : essai de méthode. Paris : Larousse, 1966b.

- La description de la signification et la mythologie comparé. L’Homme, n. 51-63, [rééd. sous le titre « La mythologie comparée » in GREIMAS, A. J. Du sens. Paris, Seuil, 1970], 1963. p. 117-134. 
. Histoire et linguistique. Annales, n. 1, 1958.

. Pour une sociologie du langage. Arguments, n. 1, 1956a.

. L'actualité du saussurisme. Le français moderne, n. 3, 1956b.

GREIMAS, A. J. ; COURTÉS, J. (Org.). Sémiotique : Dictionnaire raisonné de la théorie du langage. v. 2. Paris : Hachette, 1986.

Sémiotique : Dictionnaire raisonné de la théorie du langage. v. 1. Paris : Hachette, 1979.

GREIMAS, A. J. ; FONTANILLE, J. Sémiotique des passions : des états de choses aux états d'âme . Paris : Seuil, 1991.

GREIMAS, A. J. ; LANDOWSKI, E. (Org.). Introduction à l'analyse du discours en sciences sociales. Paris : Hachette, 1979.

GREIMAS, A. J. ; ŽUKAS, S. La Lithuanie, un des pays baltes. Vilnius : Baltos lankos, 1993.

JACKSON, B. S. Making Sense in Law. Liverpool : Deborah Charles, 1999.

JOXE, A. Voyage aux sources de la guerre. Paris : Presses Universitaires de France, 1991.

KLEIN, J.-P. ; DARRAULT, I. Pour une psychiatrie de l'ellipse. Limoges : Pulim, 2007.

LANDOWSKI, E. Honoris causa. Actes Sémiotiques [KERSYTE, N. (Org.). En quête de Greimas], n. 112, 2009. Disponible sur: <http://e publications.unilim.fr/revues/as/288>. Accédé le : 18 jlt. 2015.

Les interactions risquées. Limoges : PULIM, 2005.

Passions sans nom : Essais de socio-sémiotique III.

Paris : PUF, 2004. 
. et al. (Org.). Sémiotique en jeu : A partir et autour de l'œuvre d'A. J. Greimas. Paris-Amsterdam : Hadès- Benjamins, 1987.

LEONE, M. Spiritualità digitale. Milan: Mimesis, 2014.

Métalangages néobaroques, métalangages néoclassiques. Actes Sémiotiques, n. 116, 2013. Disponible sur: <http://epublications.unilim.fr/revues/as/1421>. Accédé le : 18 jlt. 2015.

MARRONE, G. Stupidità. Milan : Bompiani, 2012.

Addio alla Natura. Turin : Einaudi, 2011.

OLIVEIRA, A. C. (Org.). As interações sensíveis. São Paulo : Estação das Letras e das Cores, 2013.

PARRET, H. Le sublime du quotidien. Paris-Amsterdam : Hadès- Benjamins, 1988.

PERRON, P. (Org.). Paris School Semiotics. Paris-Amsterdam : Hadès- Benjamins, 1989.

SVERDIOLAS, A. Gyvenimas ir galvojimas: La vie et la réflexion. Vilnius : Vyturys, 1998.

Artigo recebido em abril de 2015 e aprovado em junho de 2015.

Disponível em: http://seer.fclar.unesp.br/casa 